\title{
Une page se tourne, mais nous en écrirons d'autres avec vous
}

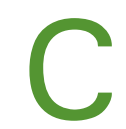

hers auteurs, lecteurs, et amis des Cahiers Agricultures,

Cette fin d'année 2013 marque un tournant dans la vie de notre revue, puisque l'Agence universitaire de la Francophonie (AUF) a décidé de mettre fin à l'appui financier qu'elle a fourni aux "Cahiers d'Etudes et de Recherche Francophones/ Agricultures " créés à son initiative il y a maintenant 20 ans.

Depuis la réunion du comité de pilotage et du comité scientifique de la revue, début mars 2013, où cette décision a été rendue publique, de nombreuses initiatives (groupes de travail, pétitions) ont été prises pour sauver le capital social et scientifique constitué au cours de ces années et trouver les moyens d'assurer la poursuite de la parution d'un titre dont l'utilité a été illustrée par l'importance de votre mobilisation.

Ces initiatives ont été fructueuses, puisque l'avenir de la revue est assuré à court terme sous une forme allégée (publication électronique), proposée par John Libbey Eurotext, qui ne remet en cause ni la ligne éditoriale ni l'équilibre entre la publication de dossiers thématiques et la publication de manuscrits soumis spontanément à la revue par des auteurs désireux d'assurer la promotion de leurs travaux, en Open Access dans une revue principalement francophone, à facteur d'impact. Les Cahiers Agricultures doivent rester une revue internationale. Il est d'ailleurs souhaitable que l'AUF continue d'assurer un parrainage de la revue et que diverses institutions d'enseignement supérieur, de recherche et de développement s'y associent, en particulier venant de pays du Sud.

Des démarches seront entreprises dans ce but au cours de l'année 2014, auxquelles tous les acteurs contribuant à la vie de la revue devront participer activement.

Le modèle économique de la publication doit encore être consolidé mais l'objectif que nous visons est bien de continuer à offrir aux chercheurs et enseignants-chercheurs la possibilité de publier leurs travaux gratuitement ou à faible coût.

Ce modèle économique ne pourra pas atteindre l'équilibre si la revue n'est pas soutenue par un panel d'institutions nationales, régionales et internationales motivées, en particulier par la publication de numéros thématiques pluridisciplinaires payants, comme celui que nous venons de publier sur les rizicultures, avec l'aide d'AfricaRice.

À l'aube de cette année nouvelle, nous souhaitons remercier chaleureusement les responsables des institutions partenaires (AUF, Inra, Cirad, IRD, Gembloux) qui nous ont permis ces dernières années de rendre la revue plus visible, mieux citée, et donc plus attractive pour les auteurs s'exprimant en français et en anglais.

Nous voudrions aussi remercier tous les évaluateurs et relecteurs qui contribuent en permanence à améliorer la lisibilité et la pertinence des manuscrits soumis à la revue.

Enfin, nous souhaitons à nouveau remercier les lecteurs, les auteurs et les amis de la revue, dont la communauté de pensée, l'intérêt et la mobilisation sont, et resteront, les éléments clés de notre pérennité et de notre reconnaissance.

Longue vie aux Cahiers Agricultures!

Le Comité de rédaction 